\title{
Pattern of road traffic fatalities in Nairobi
}

\begin{abstract}
Okemwa M.P., MBChB, MMed (Path), Lecturer, Rogena E.A., MBChB, MMed (Path), MSc (Forensic medicine), Senior Lecturer, Department of Pathology, University of Nairobi, Kenya, Rana F., MBChB, MMed (Path), Assistant Professor, Department of Pathology, Aga Khan University Hospital, Nairobi, Kenya and Gatei D.G., MBChB, FRCPath, Associate Professor, Department of Pathology, University of Nairobi, Kenya
\end{abstract}

Corresponding author: Dr. Okemwa M.P., Department of Pathology, University of Nairobi, P.O. Box 30197, Nairobi, Kenya, Email: alfaok@yahoo.com

\section{Abstract}

BACKGROUND: Road traffic injuries continue to exert a huge burden on Kenya's healthcare services. Over 134,000 crashes occur on the Kenyan roads killing more than 2,600 and seriously injuring 11,000 people annually. Injury pattern among traffic trauma admissions have been recently described while that of fatalities remains unstudied. Serious injuries will continue to result from road collisions as long as the compliance to traffic rules remains poor, the rates of seat belt use is low and the number of pedestrian walkways and flyovers few.

OBJECTIVE: To determine the pattern of fatal injuries from road traffic collisions in the city of Nairobi.

DESIGN: This was a prospective descriptive study.

SETTING: Kenyatta National Hospital (KNH) and the Nairobi City Mortuary.

SUBJECTS: One hundred consecutive road traffic fatalities autopsied between April 2003 and January 2004 (90 KNH Mausoleum, 10 Nairobi City Mortuary).

RESULTS: Casualties included 81 males and 19 females with an age range of 4-80 years and a median age of 33.5 years. Forty-five percent arrived at casualty dead while the remainder were admitted for a mean period of 14 days. The main road-user groups involved were pedestrians (62\%), passengers (24\%) and drivers $(9 \%)$. A majority $(72 \%)$ were injuries sustained along major highways.

Head trauma was the most common form of injury accounting for $76 \%$, followed by chest injuries $70 \%$, abdominal injuries $60 \%$, lower limb injuries 56\%, upper limb injuries 35\%, neck injuries $29 \%$ and pelvic injuries $24 \%$. Head, abdominal and chest injuries accounted for $57 \%$, $17 \%$ and $13 \%$ of causes of death respectively. Limb injury was the least common cause of mortality, although it may have complicated some of the other injuries.

CONCLUSION: Road traffic fatalities are predominantly a pedestrian problem. Head and trunk injuries account for $87 \%$ of the deaths. Provision of pedestrian walkways and flyovers along major highways may protect the vulnerablepedestrian population. Improvement of immediate emergency services able to cope with head and trunk injuries is recommended.

\section{Introduction}

The distribution of injuries seen on the body during autopsy can be classified into skeletal (skull, spine, limb, pelvis) and principal visceral (brain, lungs, aorta, heart, liver, spleen and kidneys) injuries. Death from these traumatic injuries follows a trimodal pattern - immediate, early and late. Immediate deaths follow injuries 
to the heart, great vessels, spinal cord and the brain. In this group, prevention rather than resuscitation and early care, is key. Early death refers to fatality within a few hours of the injury and is attributable to conditions in which immediate resuscitation and surgery would prevent death. Late deaths appear days or weeks after injury. Majority of these are secondary to sepsis and organ failure. Good critical care of injuries reduce these late deaths. Overall, 40\% of trauma deaths are preventable (1).

Data on road traffic crash characteristics in Kenya is accumulating $(2,3,4)$. Current interventions are sporadic, uncoordinated and ineffective (4). More than $75 \%$ of road traffic casualties are economically productive young adults. Pedestrians and passengers are the most vulnerable; they account for $80 \%$ of the deaths (5). Passenger-carrying vehicles particularly buses and matatus (popular public service minibuses) are the vehicles most frequently involved in fatal crashes. Current estimates indicate annual rates of road collisions, mortality and severe injuries due to road collisions to be $134,000,2,600$ and 11,000 respectively (6).

In Western literature the commonest causes of death include head injury $60 \%$, thoracic injury $20-25 \%$, and aortic injury $15 \%$. In addition, tracheobronchial injuries contribute to a small number of patients admitted to trauma centers while the spleen is the most commonly injured visceral organ (7). A Kenyan study by Odero (2) revealed that vehicle-pedestrian collisions were most severe and had a case fatality rate of $24 \%$ while only $12 \%$ of injuries resulting from vehicle -vehicle accidents were fatal. At the Kenyatta National hospital, fractures of the long bones and pelvis were the most common injuries amongst admissions but head injury predominated amongst the fatalities (4). The present post-mortem study clarifies further the pattern of injuries among road traffic fatalities at two Nairobi facilities.

\section{Materials and Methods}

The study was conducted at the Kenyatta National Hospital and Nairobi City Mortuaries. One hundred consecutive autopsies for casualties dying after road traffic crashes were evaluated between April 2003 - January 2004. Ninety autopsies were conducted at the $\mathrm{KNH}$ Mausoleum while ten were performed at the Nairobi City Mortuary (NCM). The latter 10 were all direct admissions from the scenes of accidents.

Data on the victims' demographics and circumstances of collision were obtained from the police narration as recorded in police autopsy Form 23A (Kenyan statutory postmortem form) and manually abstracted. Data from the autopsy specific to this study that included anatomical region of injury, injury severity score, histological findings in the principal visceral organs and cause of death were then transferred onto coded proforma sheets.

Computer generated spreadsheets were prepared from the database and then transferred to EPI-Info 3.2.2 $\square$ statistical software for analysis. Descriptive summary statistics were computed and the results presented in form of tables, charts and graphs.

Major highways constituted entry/exit roads from the city and busy roads outside Nairobi.

Death due to traffic accidents in Kenya is legally defined as that which occurs within one year and one day as a result of injuries sustained from that accident. In this study however, all the patients who died following traffic collisions were recruited.

\section{Results}

One hundred cases were enrolled in the study, 81 males and 19 females. The age range was 4 to 80 years with a mean age of 34.7 years (median 33.5 years). The peak ages were $30-44$ and 15-29 years (Figure 1). The ratio of those within the productive age group (15-49 years) compared to those outside this age bracket was $4: 1$. The proportion of males within this productive age group was higher $(86.4 \%$ versus $52.6 \%)$.

Majority (62\%) were pedestrians. Passengers and drivers comprised $24 \%$ and $13 \%$ of fatalities respectively (Table 1$)$.

Most collisions (72\%) occurred along major highways. Fatal collisions on roads within residential areas were $16 \%$, Central Business 
District $4 \%$, other roads $8 \%$.

A profile of the vehicles involved showed that matatus caused $38 \%$ of the collisions followed by saloon cars $17 \%$ (Figure 2). As shown in Figure 2, hit and run vehicles were involved in 19\% of accidents and by completion of the study they had not been identified hence classified as unknown.

Majority of the patients sustained multiple injuries. Most patients sustained head injuries (76\%). The proportions of patients sustaining chest, abdominal, lower extremity, neck and pelvic injuries were $70 \%, 60 \%, 57 \%, 35 \%, 29 \%$ and $24 \%$ respectively.

The injury severity score ranged from 1775 with a mean of 54.6 (median of 50). During hospitalization and treatment some developed secondary events that also contributed to their deaths.

Some organs were also found to have incidental disease processes not directly related to the collision including schistomiasis and tuberculosis.

Fifty six percent of mortalities were due to head injuries (Figure 3). The commonest form of head injury was subdural haemorrhage (50\%) followed by brain contusions $(28.9 \%)$ and brain lacerations $(21.1 \%)$. Others included subarachnoid haemorrhage (15.8\%), brain oedema $(15.8 \%)$, diffuse axonal injury $(13.9 \%)$, extradural haematoma $(6.6 \%)$, intracerebral haemorrhage $(6.6 \%)$, evisceration $(6.6 \%)$ and

\section{Figure 1: Road Traffic deaths by gender and age group, KNH 2003-2004}

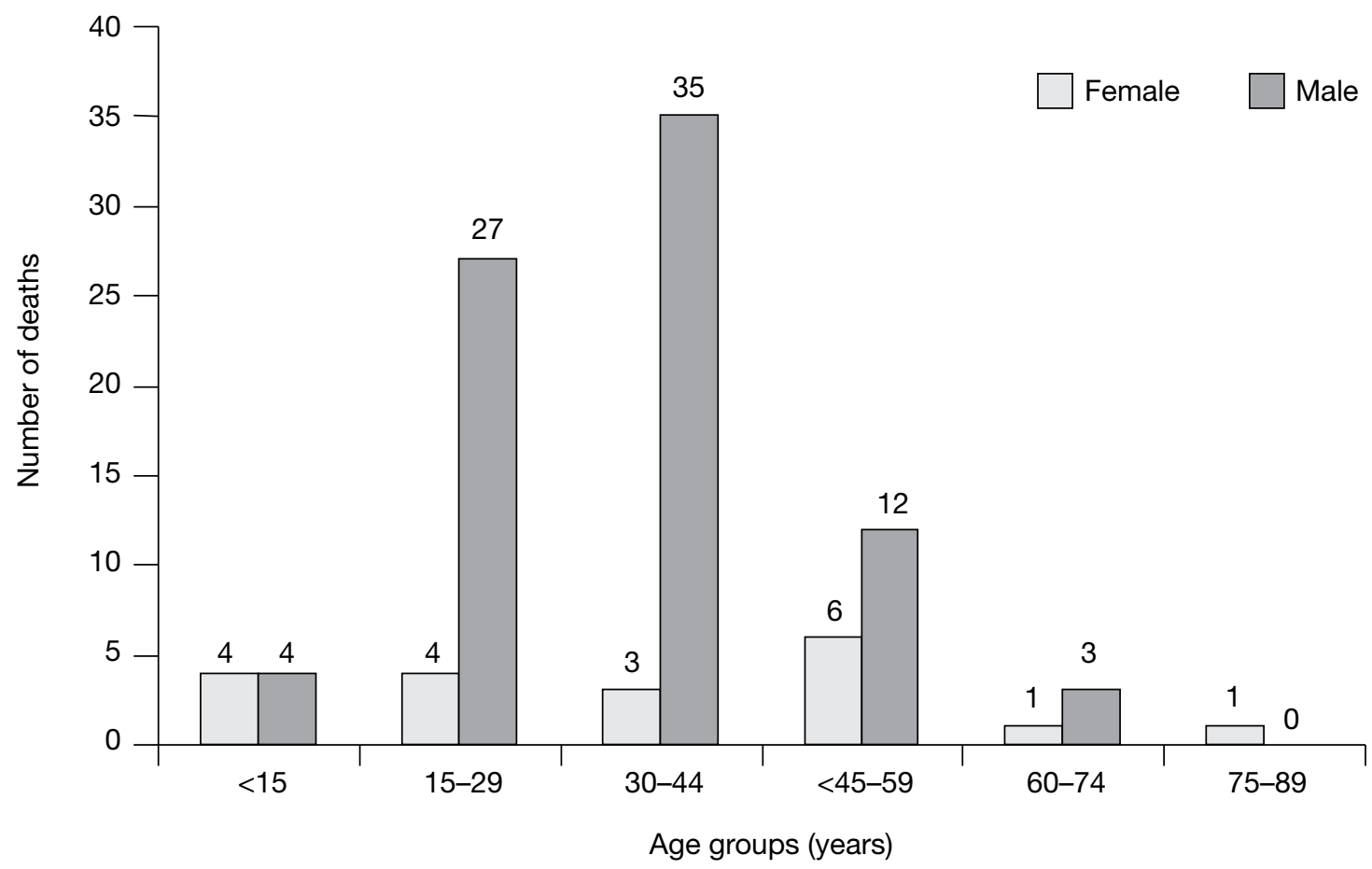

Table 1: Distribution of road user groups by gender

\begin{tabular}{lcc}
\hline Road user & Male & Female \\
\hline Pedestrian & $50(61.7 \%)$ & $12(69.3 \%)$ \\
Passenger & $17(21 \%)$ & $7(30.7 \%)$ \\
Driver & $9(16.1 \%)$ & 0 \\
Cyclists & 4 & 0 \\
Unspecified & 1 & 0 \\
\hline TOTAL & 81 & 19 \\
\hline
\end{tabular}


Figure 2: Road traffic deaths by type of vehicles

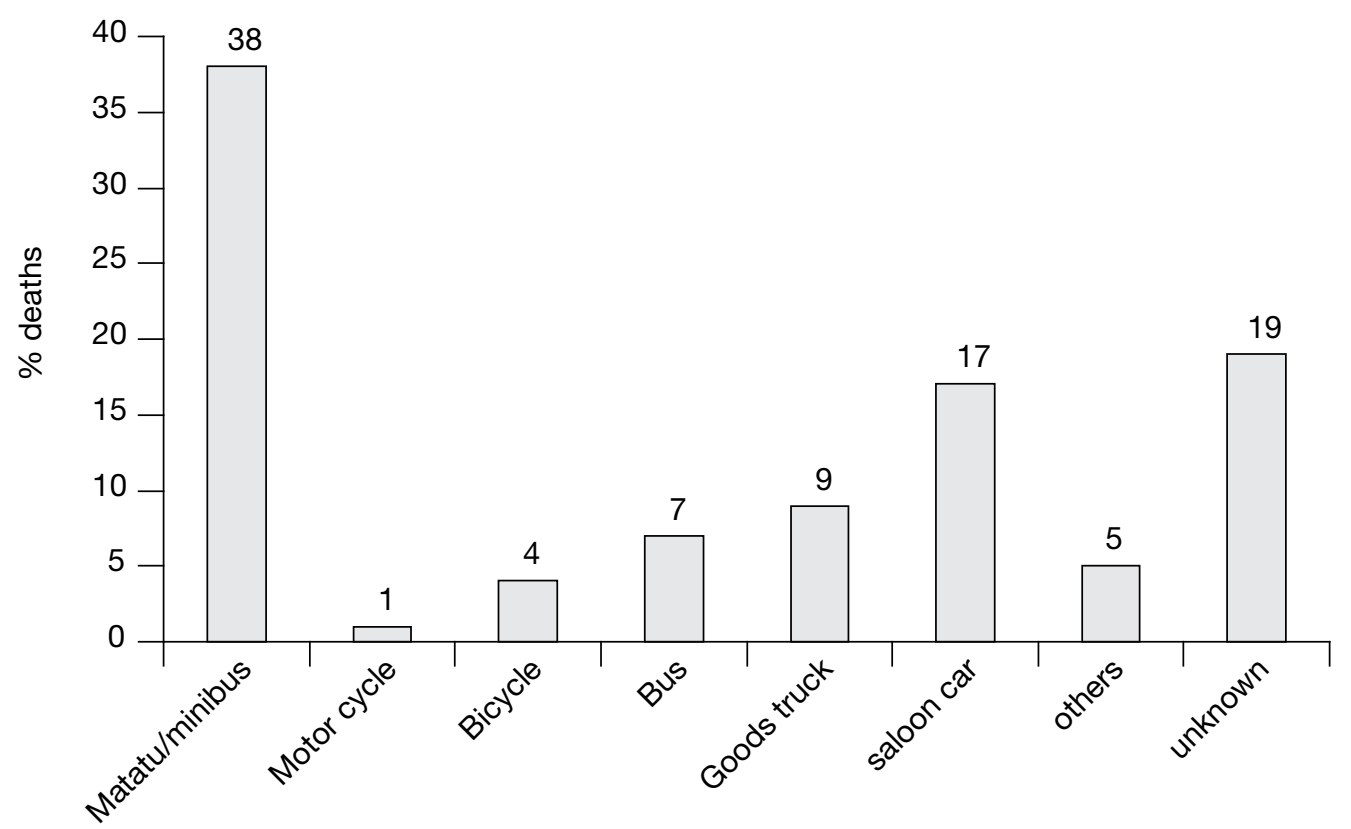

Type of vehicle

Figure 3: Cause of death by systems

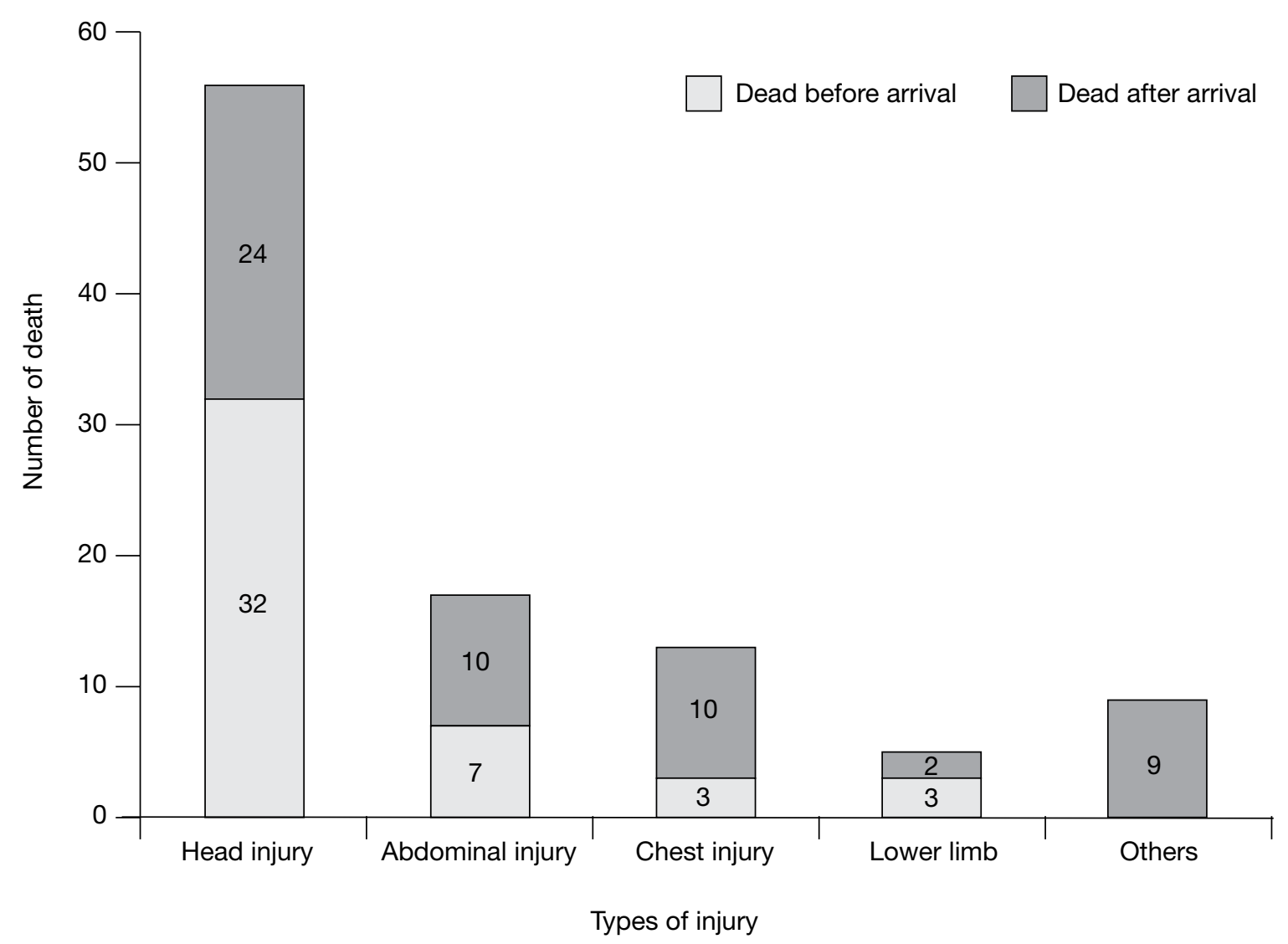


intraventricular haemorrhage (5.3\%). When the region (Figure 3) of injury was analyzed in relation to the timing of mortality, $57.1 \%$ of people who died of head injury arrived to the casualty already dead or died in casualty in comparison to $27.9 \%$ for those without head injury (OR 3.4, $95 \%$ CI $1.4-8.9, \mathrm{p}=0.004$ )

Thirteen percent of all mortalities were due to chest injuries. Rib fractures accounted for $48.6 \%$ followed by haemothorax $42.9 \%$ and pulmonary edema $31.4 \%$. The commonest secondary pathology in the chest was infection. Hypostatic bronchopneumonia (10 cases) was leading seen mainly in those admitted to ICU due to head injury. This was followed by pulmonary thromboembolism seen in four cases. Other events included aspiration ( 2 cases) and ARDS (3 cases).

Abdominal injuries were the cause of death in 17 cases. Of these, the liver was the most commonly injured organ $(46.7 \%)$ followed by the mesentery and intestines $44.9 \%$, peritoneum (haemoperitoneum, peritonitis, retroperitoneal haemorrhage) $20 \%$, spleen $10 \%$ and kidney $6.7 \%$.

The proportion of deaths attributable to limb injuries was $5 \%$. The leading pelvic injuries were fractures $(50 \%)$ and perivesical haematomas $(20.8 \%)$. Other injuries included urinary bladder contusions (4 cases), ruptured bladder (3 cases) and testicular contusions ( 2 cases). Decubitus bed sore was the commonest secondary event seen in $25 \%$ of the pelvic injuries. The earliest decubitus pressure sore was recorded at 21 days after hospitalization and inevitably seen after 90 days.

The femur was affected in $47.4 \%$ of the lower limb injuries followed by tibia/fibula fractures $(36.9 \%)$. In the upper limb, humeral fractures were the most common (31.4\%) followed by radioulnar fractures $14.3 \%$.

\section{Discussion}

The results of the present study indicate male prevalence of $80 \%$. This finding is similar to the study by Odero et al, which showed that more than $75 \%$ of road traffic casualties were males in the economically productive age group
(2). Similarly, the pedestrian and passenger vulnerability corroborates the data by Odero et al. The road user involvement was different for the two genders reflecting differences in socioeconomic engagements. About $31 \%$ of the female subjects were passengers compared to $21 \%$ of the males. Equally revealing was the result of no female drivers compared to the 13 male drivers (Table 1).

Head injuries caused the highest fatality. This result is consistent with other data (4). Among admitted road traffic trauma patients at the Kenyatta National Hospital, Nairobi, fractures contributed to $48.4 \%$ of the total injuries recorded while head injuries contributed only $22.8 \%$ (4) but among fatalities, $75 \%$ were due to head injury (4). That majority of patients sustaining head injuries will arrive dead as compared to other regional injuries is also not surprising. The country's pre-hospital care systems should therefore recognize the important place of severe head injuries in contributing to trauma mortality. A finding also related to the emergency care of injured patients is the prevalence of neck injuries. Twenty nine percent of all cases had neck injuries. These injuries may be seen in pedestrians or vehicle occupants who have been ejected - as a result of the head violently striking the road surfaces or solid objects (8). Head injury amongst pedestrians has been attributed mainly to speeding vehicles, lack of walkways and flyovers for pedestrians and drunkenness in crashes occurring in the evening and night hours (8).

In cases involving the abdomen, the liver was the most commonly involved organ. This vulnerability is due to its big solid size, fixation and anterior placement compared to kidneys and spleens which are located in the paravertebral gutters and thus protected by muscle and vertebral column. These underlying visceral organ pathologies may be responsible for causing collisions in a minority of cases (9).

Decubitus bed sores were a common secondary event in pelvic injury. In this study, the earliest decubitus pressure sore was recorded at 21 days after hospitalization and inevitably seen after 90 days. These sores result from lying in one position for long especially on bony prominences and may indicate inadequate 
quality of nursing.

Severity and critical injuries based on ISS are predicted to have higher mortality (10). In this study, the score ranged from 17- 75 with a mean of 54.6. Severe and critical injuries based ISS predict higher levels of mortality. In our study, patients with all levels of ISS died. The reasons for the avoidable mortality need to be explored.

In conclusion, this study has documented a predominant pedestrian vulnerability and head involvement in road trauma fatality. Traffic laws, especially on major highways, should be enforced. More focus to protect pedestrians should be undertaken to provide walkways, cycling ways and flyovers along these highways.

\section{References}

1. Meyer A.A. Death and disability from injury: A global challenge. J. Trauma. 1998; 44: 1-12.

2. Odero W. Road traffic accidents In Kenya; An epidemiological appraisal: East Afr. Med. J. 1995; 72: 299-305.

3. Saidi H.S. and Kahoro P. Experience with road traffic accident victims at the Nairobi Hospital: East Afr. Med. J. 2001; 78: 441-444.

4. Muyembe V.M. Patterns of injuries from traffic accidents seen in emergency admissions at $\mathrm{KNH}$ : Thesis, University of Nairobi, 1992.

5. Odero W., Khayesi M. and Heda P.M. Road traffic injuries in Kenya: Injury Control Safety Promotion. 2003; 10(1-2): 53-61.

6. The Daily Nation. Wednesday, February 18, 2004. Pg 19.

7. Bowley D. and Bafford K. Pattern of injury in motor vehicle accidents, Oct. 2002.

8. Knight B. Simpson's textbook of forensic medicine, London Arnold, 1997; 11: 80-83.

9. Anthony P.P. and Macsween M.N.R. Recent advances in Histopathology. 11: 232-233

10. Baker S.P., O'Neill B., Hadon W., et al. The Injury Severity Score: a method for describing patients with multiple injuries and evaluating emergency care. J. Trauma. 1974; 14: 187-196 